Palabra Clave (La Plata), abril 2017, vol. 6, n², e018. ISSN 1853-9912

Universidad Nacional de La Plata.

Facultad de Humanidades y Ciencias de la Educación.

Departamento de Bibliotecología

\title{
Prueba piloto de implementación del estándar de catalogación RDA en Colombia
}

\author{
Pilot test for the implementation of RDA cataloging \\ standard in Colombia
}

\section{Fabio Ernesto Tuso González*}

* Biblioteca Nacional de Colombia. Grupo de Procesos Técnicos, Colombia | ftuso@bibliotecanacional.gov.co

\section{PALABRAS CLAVE}

Catalogación

Metadatos

Bibliotecas digitales, Recursos electrónicos

Sistemas Integrales de Bibliotecas (SIB)

RDA

FRBR

Tecnología en servicios técnicos

\section{RESUMEN}

La Biblioteca Nacional de Colombia, como ente rector en la formulación, coordinación y ejecución de políticas, planes y programas relacionados con el procesamiento técnico del patrimonio bibliográfico y documental colombiano, lideró la prueba piloto de implementación del estándar de catalogación RDA en Colombia. Esta prueba piloto, cuenta actualmente con la participación de diferentes unidades de información del país como la Biblioteca Luis Ángel Arango, la Biblioteca Pública Piloto de Medellín, el Instituto Caro y Cuervo, el SENA, Corpoica, la Corporación Biblioteca Rafael Carrillo Luquez, la Fundación Universitaria de Ciencias de la Salud FUCS (Fundación Universitaria Ciencias de la Salud, la Universidad de Antioquia, la Universidad de " El Bosque", la Universidad del Valle, la Universidad Javeriana, la Universidad Nacional de Colombia (Manizales), la Universidad Pontificia Bolivariana y la Universidad Santo Tomás, todas ellas interesadas en estudiar la norma y establecer políticas de aplicación de forma consensuada, además de reconocer los cambios e impactos de su implementación. En este sentido, para la prueba piloto RDA se han efectuado reuniones periódicas, tanto presenciales como virtuales, con el objetivo de trabajar en la creación de registros RDA, los cuales son aplicables a todo tipo de recursos y contenidos, en especial a los del entorno digital. La experiencia de esta prueba piloto representa un referente para la implementación de la norma RDA en América Latina, dado que ha generado diversos productos: adaptación de software libre y configuración de los SIB (Sistema Integrado de Bibliotecas) de las entidades participantes para el modelo de metadatos, etiquetas y campos fijos MARC21 necesarios para la elaboración de registros RDA, elaboración de registros (obra, expresión, manifestación, autoridad: personal, corporativo y de familia), creación de un foro especializado para intercambiar, publicar, poner al servicio avances y, finalmente, elaborar manuales relacionados con la creación de registros. Considerando la importancia de esta prueba piloto para América Latina, este artículo presenta todos los aspectos relevantes de su desarrollo, los resultados obtenidos y los pasos a seguir en el futuro.

\section{KEYWORDS ABSTRACT}

Cataloging

Metadata

Digital libraries

Electronic resources

Integrated Library Systems

FRBR

Technology in technical services
The National Library of Colombia, as the guiding agency in the formulation, coordination and execution of policies, plans and programs related to technical processing of Colombian bibliographic and documentary heritage, led the pilot test for implementing of the RDA cataloging standard in Colombia. This pilot test, currently has the participation of different information units in the country, such as the Luis Ángel Arango Library, the Pilot Public Library of Medellín, the Caro and Cuervo Institute, the SENA, Corpoica, the Rafael Carrillo Luquez Library Corporation, the University Foundation of Health Sciences, the University of Antioquía, the University of "El Bosque", The University of "Del Valle", the Javeriana University, the National University of Colombia (Manizales), the "Pontificia Bolivariana" University, and the "Santo Tomás" University, all of them interested in studying the norm and establishing implementation policies in a consensual way, in addition to recognize the changes and impacts of its implementation. In this sense, for the RDA pilot test, there have been carried out meetings, both face-to-face and virtual, with the objective of working on RDA records creation, to be applicable to all resources and content types, especially to those coming from the digital environment. The experience of this pilot test represents a reference for the implementation of RDA standard in Latin America, to the extent that it has generated some outcomes: free software adaptation, as well as the configuration of ILS (Integrated Library System) of participating entities for the metadata model, MARC fields and fixed-length data elements, that are necessary for the creation of RDA records (work, expression, manifestation, as well as personal, corporate and family authorities); establishment of a specialized forum to exchange, publish, make advances, and finally, develop manuals related to records creation. Considering the importance of this pilot test for Latin America, the article shows all relevant aspects of its development, the results obtained and the steps to be taken in the future.

Recibido: 15 de diciembre de 2016 | Aceptado: 28 de febrero de 2017 | Publicado: 20 de abril de 2017 
Sin duda alguna, los grandes avances tecnológicos y las nuevas maneras de desarrollar catálogos y la organización de colecciones, en torno a la integración de contenidos para las unidades de información, tanto en soportes físicos como digitales, frente a la integración única de recursos, ha motivado nuevos enfoques en la manera de representar la información. Junto a esto, la bibliotecología, a partir del avance y desarrollo del nuevo estándar de descripción de contenido RDA (Recursos, Descripción y Acceso), conceptualmente ha replanteado un nuevo enfoque en la manera de registrar, describir y presentar la información bibliográfica para las unidades de información y sus catálogos.

En este sentido, la Biblioteca Nacional de Colombia (BNC) lidera, como ente rector en la formulación, coordinación y ejecución de políticas, planes y programas relacionados con el procesamiento técnico del patrimonio bibliográfico y documental colombiano, la prueba piloto de implementación del estándar de catalogación RDA en Colombia.

La BNC aplica el estándar de catalogación RDA (Resources: Description and Access/ Recursos: Descripción y Acceso) en la catalogación de los objetos digitales como una nueva norma de contenido, reemplazando a la RCAA2. De esta manera, brinda características novedosas que ofrecen otras posibilidades de catalogación y que apoyan la creación de datos bien estructurados, mejora la experiencia de los usuarios en los entornos de búsqueda y recuperación de información, y facilita -a su vez- la consulta de recursos bibliográficos en los ambientes digitales actuales; por ejemplo: permite que una entrada del catálogo, correspondiente a un material bibliográfico, agrupe más posibilidades de búsqueda, recuperación y acceso a la información que en el estándar anterior.

Esta prueba piloto cuenta con la participación de diferentes unidades de información del país como la Biblioteca Luis Ángel Arango, la Biblioteca Pública Piloto de Medellín, el Instituto Caro y Cuervo, el SENA, Corpoica, la Corporación Biblioteca Rafael Carrillo Luquez, la Fundación Universitaria de Ciencias de la Salud FUCS, la Universidad de Antioquia, la Universidad de "El Bosque", la Universidad del Valle, la Universidad Javeriana, la Universidad Nacional de Colombia (Manizales), la Universidad Pontificia Bolivariana y la Universidad Santo Tomás, que están interesadas en estudiar la norma y establecer políticas de aplicación de forma consensuada, además de reconocer los cambios e impactos de su implementación. En este sentido, la prueba piloto RDA ha celebrado reuniones periódicas tanto presenciales como virtuales desde el 26 de marzo de 2015, con el objetivo de trabajar en la creación de registros RDA, los cuales son aplicables a todo tipo de recursos y contenidos, en especial los del entorno digital.

La prueba está generando avances a partir de las herramientas que brinda la norma para su implementación, tales como un plan de lectura del RDA Toolkit (RDA Steering Committee, 2010) y los modelos conceptuales FRBR (Requisitos Funcionales de los Registros Bibliográficos) (Tillet, 2005) y FRAD (Requisitos funcionales de los Datos de Autoridad) (IFLA, 2008), así como un cronograma de elaboración de registros (obra, expresión, manifestación, autoridades de familia, corporativo y personal). Promueve, además, un intercambio permanente de experiencias e inquietudes sobre la implementación de este nuevo estándar de catalogación.

La experiencia de esta prueba piloto representa un referente para la implementación de la norma RDA en América Latina, dado que ha generado diversos productos: adaptación de software libre y configuración de los SIB (Sistema Integrado de Bibliotecas) de las entidades participantes para el modelo de metadatos; etiquetas y campos fijos MARC21 necesarios para la elaboración de registros RDA; elaboración de registros (obra, expresión, manifestación, 
autoridad: personal, corporativo y de familia); creación de un foro especializado para intercambiar, publicar, poner al servicio avances y finalmente: elaborar manuales relacionados con la creación de registros. Considerando la importancia de esta prueba piloto para América Latina, este artículo presenta todos los aspectos relevantes de su desarrollo, los resultados obtenidos y los pasos a seguir en el futuro.

Con miras al 2017, se espera adelantar la publicación electrónica de guías metodológicas de trabajo para la catalogación de objetos específicos, como: mapas, partituras, libros impresos y digitales, manuscritos, publicaciones seriadas y objetos digitales, entre otros. Asimismo, se espera promover el desarrollo de cursos e-learning y políticas con el objetivo de apoyar al sector bibliotecario en el avance de los estándares de catalogación para el país.

La Biblioteca Nacional de Colombia (2015), mediante el FORO RDA ha desarrollado modelos, guías y plantillas para la elaboración de registros con el estándar RDA de diferentes tipos: Obra, Expresión, Manifestación (audiovisual, mapas, partitura, pagina web, e-libro, etc.), autoridad personal, autoridades cooperativa y de familia. Asimismo, las principales características y apuestas del FORO/RDA son presentadas en la Tabla 1 , el cual puede consultarse en la siguiente dirección: http://bibliotecanacional.gov.co/RDA/foro:

Tabla 1. Principales características y apuestas del FORO/RDA

Brindar acceso a los contenidos desarrollados en la prueba piloto de manera abierta, libre y accesible.

Propender a la colaboración bibliotecaria local e internacional.

Generar mecanismos de debate frente al desarrollo de las temáticas orientadas a RDA.

Brindar cercanía y discusión con los participantes y usuarios en general.

Continuidad y seguimiento en las discusiones y temáticas.

Brindar temáticas particulares en el desarrollo de contenidos RDA (guías, actas, manuales, archivos de consulta y presentaciones, entre otros).

Búsquedas generales y específicas en los contendidos y tópicos.

Acceso 7/24 a la información y los contenidos desarrollados en la prueba piloto.

Seguimiento al número de consultas y participantes.

Brindar libre opinión y expresión a todos los interesados participantes. 
Por otro lado, algunos de los registros RDA, a partir de la metodología planteada desde el 2015 en el FORO/RDA, son presentados a continuación:

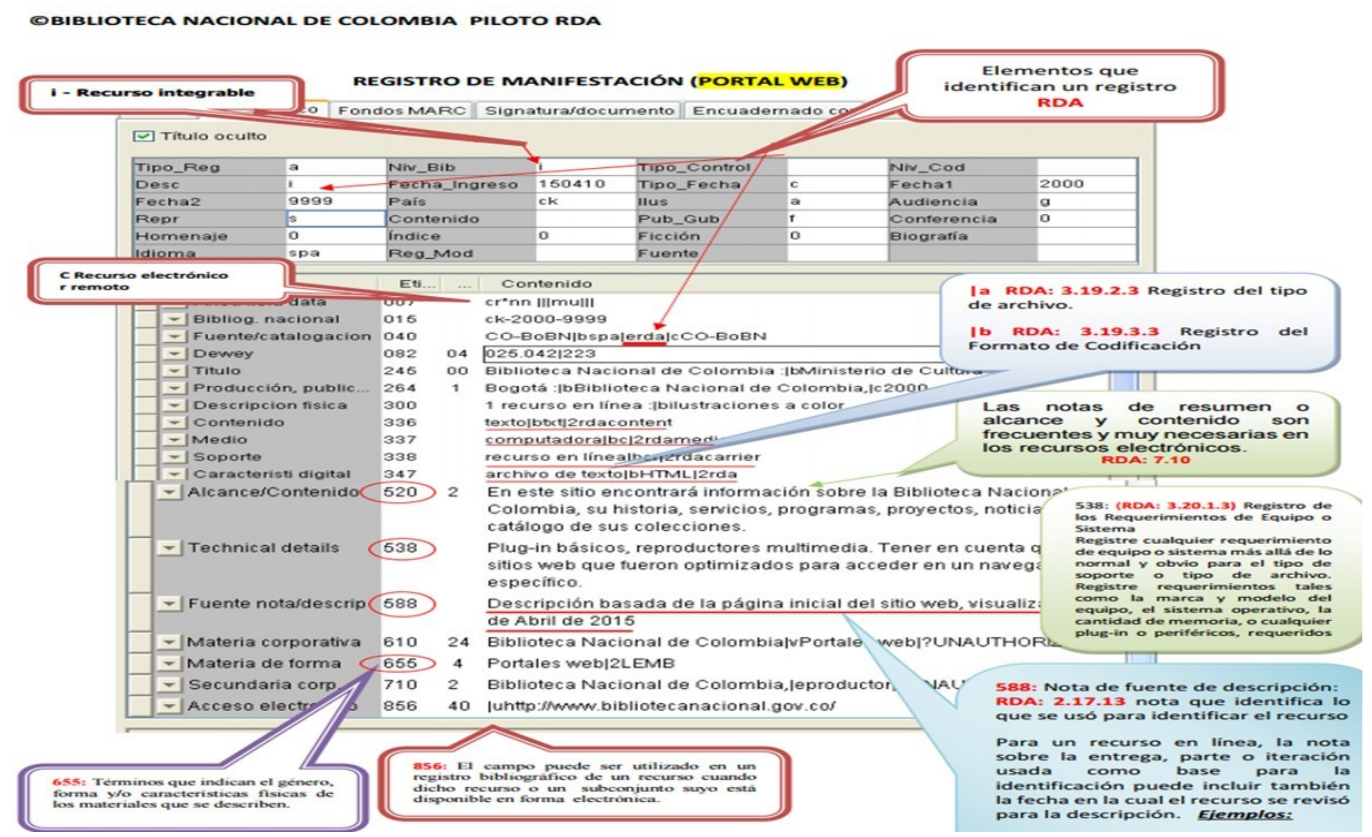

Figura 1. Registro de manifestación RDA/BNC

El registro de la Figura 1, accesible mediante el FORO/RDA, corresponde a una manifestación (Portal Web) en la cual es posible identificar las etiquetas necesarias para la correcta generación de registros para este tipo de manifestaciones, aplicando la norma RDA a partir del RDA Toolkit. En el registro se incluyen:

- Campos fijos necesarios para la correcta identificación de un registro RDA.

- Correcta identificación de la fuente de catalogación.

- Etiquetas 336, 337, 338 y 347 relacionadas con la correcta identificación del contenido, medio, soporte y características digitales.

- Etiquetas 520 y 538, notas de alcances de contenido y detalles técnicos.

- Etiqueta 588, Fuente / Descripción.

- Etiqueta 655, Materia de forma y/o características físicas de los materiales. 


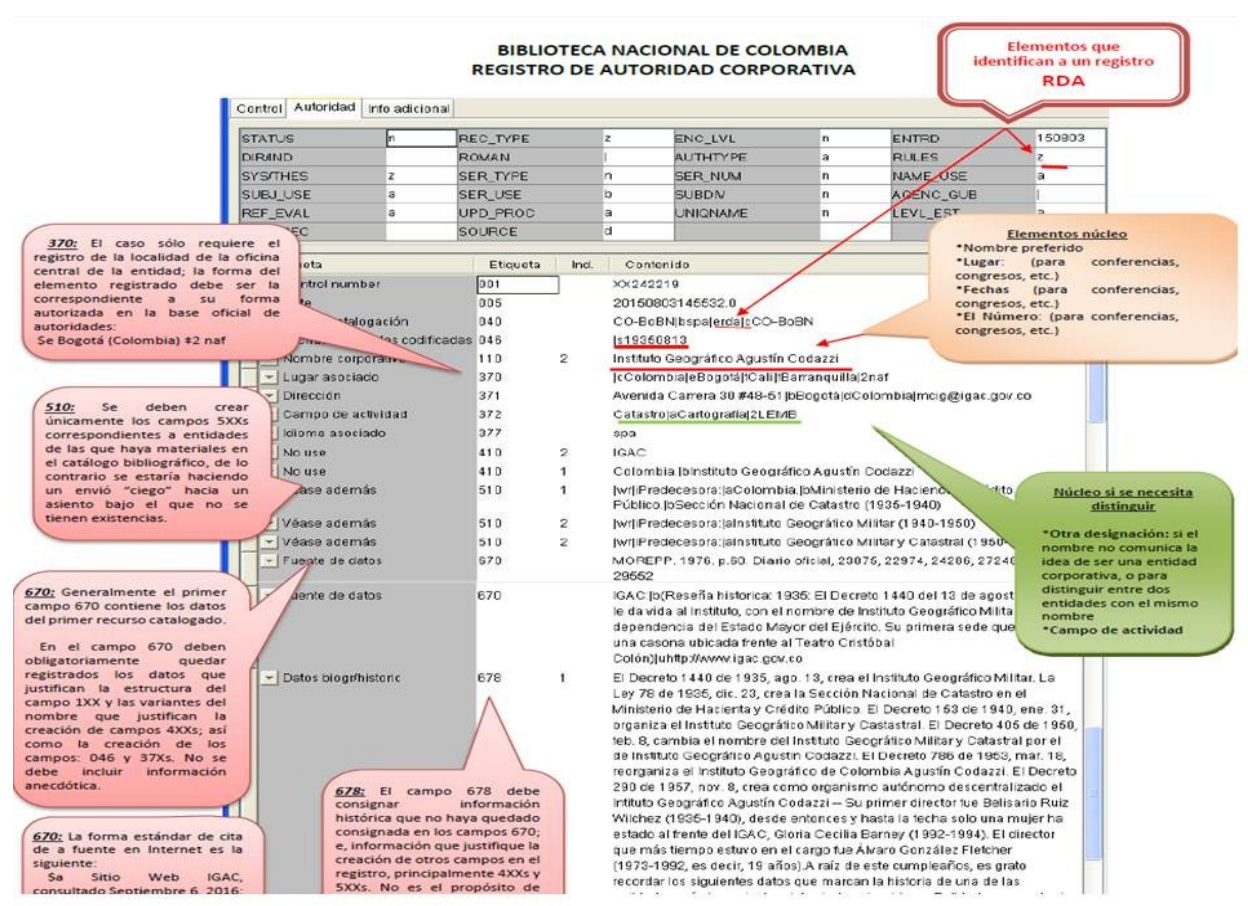

Figura 2. Registro de autoridad corporativa RDA/BNC

En el caso de la Figura 2, correspondiente a un registro de autoridad, es posible identificar las etiquetas y aplicación del estándar RDA, en donde se incluyen anotaciones referentes a cada una de ellas:

- Campos fijos necesarios para la correcta identificación de un registro RDA.

- Correcta identificación de la fuente de catalogación.

- 370, lugar asociado.

- 371, dirección.

- 372 , campo de actividad.

- 377 , idioma.

- 410, no use.

- 510, véase además.

- 670, fuente de datos.

- 678 , datos bibliográficos.

Las entidades y especialistas interesados en este tema pueden encontrar en el sitio web del Foro/RDA (http://bibliotecanacional.gov.co/RDA/foro), que se muestra en la Figura 3 , los avances, actas, registros, documentos, manuales de apoyo y enlaces de interés, así como la metodología, los objetivos y los alcances de la prueba. 


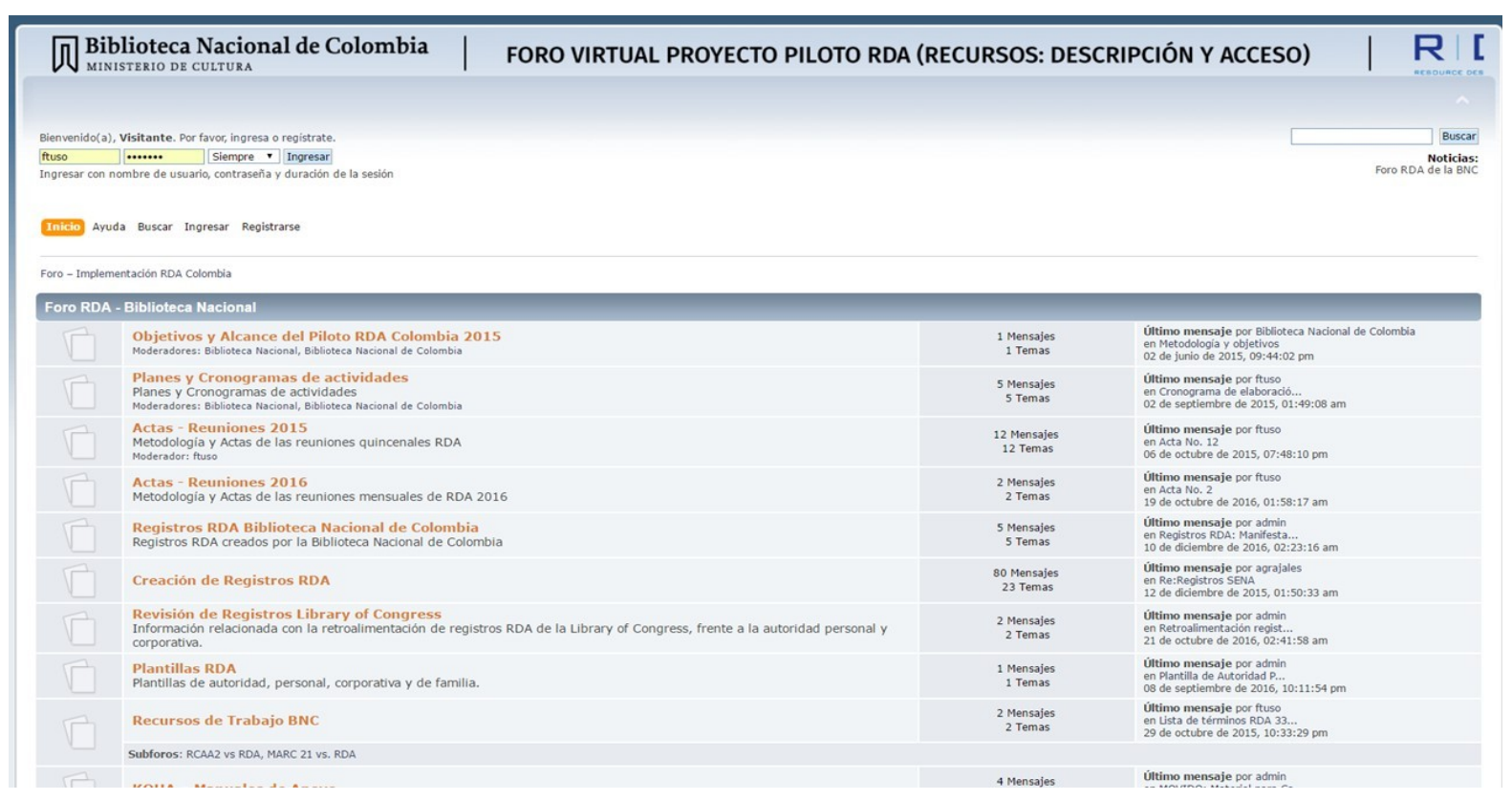

Figura 3. FORO/ RDA Biblioteca Nacional de Colombia

Los resultados de la implementación del estándar desde 2015 en 25 unidades de información, entre las que se encuentran bibliotecas universitarias, públicas y especializadas, ha permitido identificar los siguientes aspectos:

1. No todo el software bibliográfico está preparado para el cambio y aplicación del estándar RDA.

2. El desarrollo de los modelos conceptuales (FRBR/Requisitos Funcionales de los Registros Bibliográficos - FRAD/ Requisitos funcionales de los Datos de Autoridad), no pueden ser aplicables de acuerdo al diseño y desarrollo del software actual.

3. Es necesario repensar y promover que el sector bibliotecario se involucre en el desarrollo de la implementación del modelo RDA de la mano con la industria del software bibliográfico para el desarrollo de estos cambios y la configuración precisa de los SIB (Sistema Integrado de Bibliotecas).

4. En su mayoría, los proveedores de software desconocen cómo aplicar el estándar y los cambios de RDA.

5. Las solicitudes de algunos cambios de las etiquetas, de acuerdo a lo necesario para aplicar el estándar RDA, no pueden ser respondidas por algunos proveedores.

6. Al diligenciar los registros de obra, expresión y manifestación, no existen relaciones entre ellos, tal como pretende el modelo.

7. Los despliegues de la información en MARC dentro de los OPAC, no permiten visibilizar correctamente la información.

8. Los cambios desarrollados por RDA en la descripción de los registros son favorables para los usuarios, facilitando la no abreviación de información y una mejor recuperación de los registros. 
9. La elaboración de registros RDA es mucho más dispendiosa por la complejidad y desarrollo descriptivo que indica la norma.

10. La adopción del estándar para un nivel de descripción amplio requiere un mayor número de tiempo y recursos humanos.

Las principales dificultades de las entidades participantes en la prueba piloto y de la propia Biblioteca Nacional de Colombia, han sido la correcta implementación de los modelos RFDR/FRAD (fundamento del estándar RDA) en las herramientas de software bibliográfico. El desarrollo actual de éstos no permite desplegar el modelo conceptual, ni tampoco integrarlo en la recuperación de los datos, de acuerdo al planteamiento de Tillet (2005), presentado en la Figura 4.

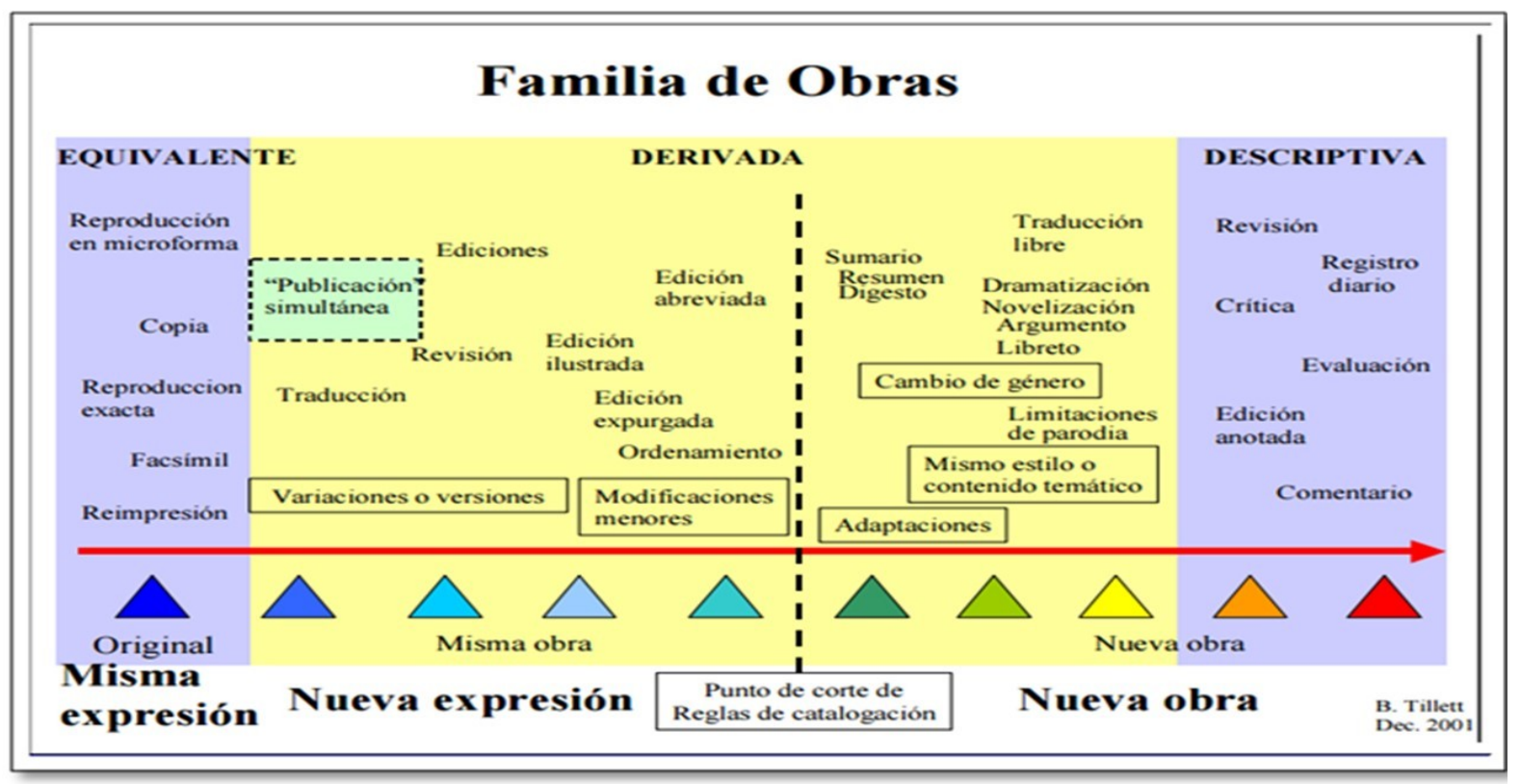

Figura 4. Relaciones Bibliográficas, Tillet (2005).

En este sentido, hasta que los grupos planteados en los modelos conceptuales RFDR/FRAD no sean visibles en los catálogos bibliográficos y/o las relaciones no se desplieguen para los usuarios de las unidades de información y no sean repensados de acuerdo con la correcta implementación de RDA, podríamos concluir que ninguna entidad puede implementar al $100 \%$ el estándar RDA, puesto que el software bibliográfico actual no lo permite. Para esto es necesario realizar algunos desarrollos en los OPAC. Los desarrolladores de software bibliográfico deben considerar este modelo conceptual y los SIBs, tanto comerciales como libres, deben avanzar en estos desarrollos. En este sentido, aplicar RDA no es etiquetar, o adicionar campos y subcampos MARC, sino que la correcta aplicación debe responder a una nueva manera de representación de los datos bibliográficos y registros funcionales.

Finamente, cabe precisar y recalcar que la implementación de RDA no implica y/o se centra exclusivamente en la aplicación de las instrucciones de etiquetado o marcado en nuevas etiquetas, campos fijos e indicadores planteados por RDA, ya que esto no garantiza implementar en su totalidad el modelo, puesto que no existe una correlación de los datos en las relaciones de los registros de obra, expresión y manifestación. 


\section{Biblioteca Digital BNC}

Desde la Biblioteca Nacional estamos aplicando el estándar en la catalogación de los objetos digitales y la creación de autoridades, garantizando la aplicación del estándar RDA en los registros de Obra, Expresión, Manifestación e Ítem para todos los nuevos objetos digitales que son reproducción y/o nacidos digitales, a partir del proceso de depósito legal digital.

Las colecciones digitales de la Biblioteca Nacional de Colombia cuentan con la aplicación del estándar RDA, y están conformadas por una variedad de recursos tanto digitalizados como nacidos digitales de diferentes tipos: libros, manuscritos, prensa, revistas, dibujos, grabados, fotografía, sonido y video.

En 2009 se inició la digitalización de sus fondos patrimoniales, y desde 2012 recoge la producción nacida en digital que están entregando los productores de obras colombianas, como muestra del Depósito Voluntario de esos contenidos. Los recursos de la Biblioteca Digital se encuentran en http://catalogoenlinea.bibliotecanacional.gov.co/client/es ES/bd (Figura 5).
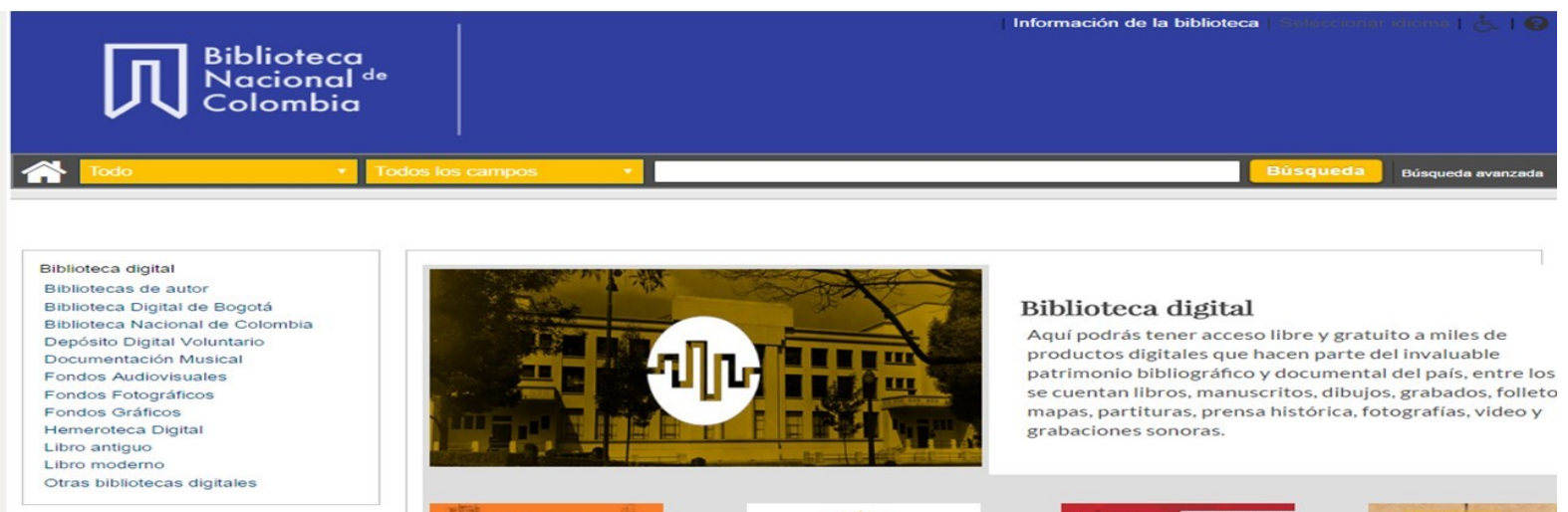

Biblioteca digital

Aqui podrás tener acceso libre y gratuito a miles de patrimonio bibliográfico y documental del país, entre los se cuentan libros, manuscritos, dibujos, grabados, folleto mapas, partituras, prensa histórica, fotografias, video y

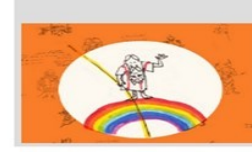

Disfruta del sexto capítulo de Historia de Colombia

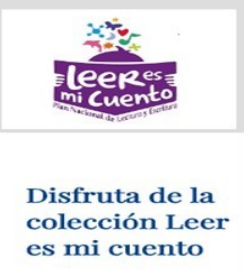

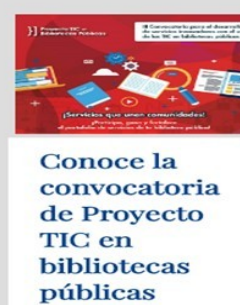

LA PIEZA DEL WV:

Descubre la pieza del mı

Figura 5. Biblioteca Digital BNC / Sirsidynix Enterprise Portfolio

Algo interesante de la puesta en marcha de la Biblioteca Digital es la migración de MARC a Dublin Core, sincronizando y mapeando los datos bibliográficos de la forma presentada en la Tabla 2.

\section{Tabla 2. MARC/RDA a Dublin Core}

\begin{tabular}{|l|l|l|}
\hline \multicolumn{1}{|c|}{ Campos MARC 21 } & \multicolumn{1}{|c|}{$\begin{array}{l}\text { Elementos } \\
\text { Dublin Core }\end{array}$} & \multicolumn{1}{|c|}{ Notas de implementación } \\
\hline 700 Autor secundario - persona. & DC: & Separar _; \\
710 Autor secundario - entidad. & Contributor & Ministerio de Cultura ; Biblioteca Nacional \\
711 Autor secundario - congreso. & & \\
\hline
\end{tabular}


Palabra Clave (La Plata), abril 2017, vol. 6, n² 2, e018. ISSN 1853-9912

\begin{tabular}{|c|c|c|}
\hline & & Sí se encuentra con más de un autor \\
\hline 651 Materia Geográfica & $\begin{array}{l}\text { DC: } \\
\text { Coverage }\end{array}$ & \\
\hline $\begin{array}{l}100 \text { Autor principal - persona. } \\
110 \text { Autor principal - entidad. } \\
111 \text { Autor principal - congreso. }\end{array}$ & DC: Creator & $\begin{array}{l}\text { Tuso González, Fabio ; Carrillo, Jenny } \\
\text { Sí se encuentra con más de un autor }\end{array}$ \\
\hline 008 Posiciones 07-10 Fecha. & DC: Date & Recomendamos la utilización de uno de los formatos \\
\hline $\begin{array}{l}260 \text { Subcampo |c Fecha } \\
\text { publicación. } \\
260 \text { Subcampo |g Fecha impresión. } \\
264 \text { Subcampo |c Fecha } \\
\text { publicación. }\end{array}$ & & $\begin{array}{l}\text { definidos en el documento "Date and Time } \\
\text { Formats", http://www.w3.org/TR/NOTE-datetime bas } \\
\text { ado en la norma ISO } 8601 \text { que incluye, entre otras, } \\
\text { fechas en el formato AAAA y AAAA-MM-DD. De esta } \\
\text { forma la fecha 1994-11-05 correspondería al } 5 \text { de } \\
\text { Noviembre de } 1994 \text {. }\end{array}$ \\
\hline $\begin{array}{l}\text { >500-599 Notas. } \\
\text { Excepto las etiquetas: } \\
506 \text { Nota de restricciones de } \\
\quad \text { acceso. } \\
530 \text { Nota del formato físico } \\
\text { adicional disponible. } \\
540 \text { Nota de condiciones de uso y } \\
\text { reproducción. } \\
546 \text { Nota de idioma. }\end{array}$ & $\begin{array}{l}\text { DC: } \\
\text { Description }\end{array}$ & $\begin{array}{l}\text { Todas las notas del } 500 \text { al } 599 \text { con las excepciones } \\
\text { descritas, que irían en DC:Rights }\end{array}$ \\
\hline $\begin{array}{l}533 \text { Medio físico. } \\
538 \text { Nota de detalles del sistema } \\
336 \text { subcampo a } \\
337 \text { subcampo a } \\
338 \text { subcampo a } \\
347 \text { Características digitales }\end{array}$ & DC: Format & \\
\hline $\begin{array}{l}020 \text { Subcampo |a ISBN. } \\
022 \text { Subcampo |a ISSN. } \\
856 \text { Subcampo |u URL. }\end{array}$ & DC: Identifier & Si se encuentra más de uno se duplica. Separar _; \\
\hline
\end{tabular}




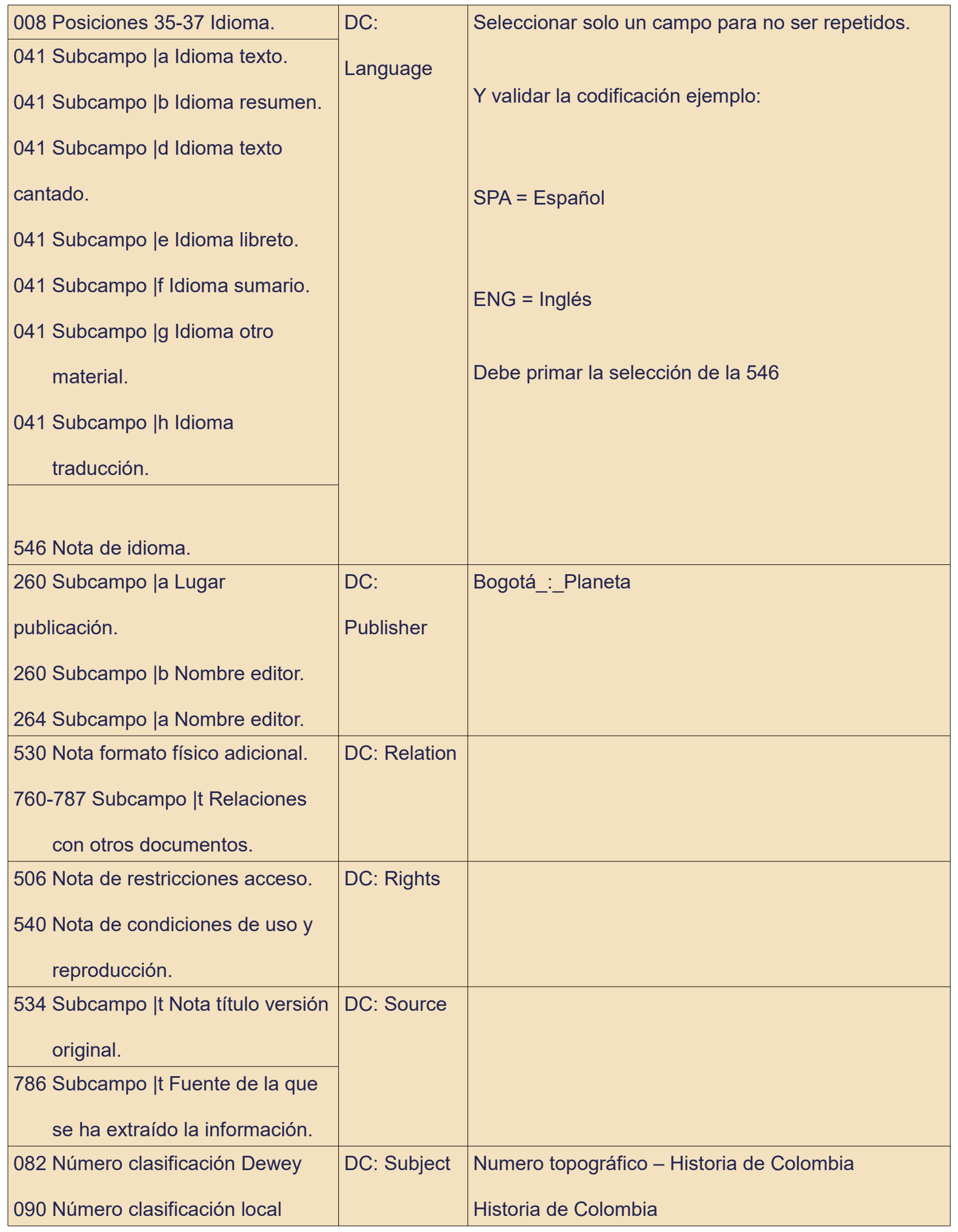




\begin{tabular}{|c|c|c|}
\hline $\begin{array}{l}600 \text { Materia - nombre persona. } \\
610 \text { Materia - nombre entidad. } \\
611 \text { Materia - nombre congreso. } \\
630 \text { Matería - título uniforme. } \\
650 \text { Materia general. } \\
653 \text { Materia - Cronológica. }\end{array}$ & & \\
\hline $\begin{array}{l}245 \text { Título propio. } \\
246 \text { Variante de título } \\
130 \text { Asiento Principal-Título } \\
\quad \text { Uniforme } \\
210 \text { Título abreviado. } \\
222 \text { Título clave. } \\
240 \text { Título uniforme. } \\
242 \text { Traducciones del título por la } \\
\text { Agencia Catalogadora. } \\
243 \text { Título uniforme colectivo. } \\
247 \text { Título anterior. }\end{array}$ & DC: Title & $\begin{array}{l}\text { El elemento DC:Title es repetible para indicar la } \\
\text { información contenida en las etiquetas: } \\
210 \text { Título abreviado. } \\
222 \text { Título clave. } \\
240 \text { Título uniforme. } \\
242 \text { Traducciones del título por la Agencia } \\
\text { Catalogadora. } \\
243 \text { Título uniforme colectivo. } \\
247 \text { Título anterior. }\end{array}$ \\
\hline $\begin{array}{l}\text { Leader posición } 06 \text { Tipo tipo de } \\
\text { registro. } \\
\text { Leader posición } 07 \mathrm{Nivel} \\
\text { bibliográfico } \\
655 \text { Materia - subencabezamiento } \\
\text { de forma. }\end{array}$ & DC: Type & \\
\hline
\end{tabular}

Para llevar a cabo lo anterior y con el objetivo de convertir los datos se utilizó MarcEdit (Figura 6 y Figura 7) como una herramienta de mapeo. 


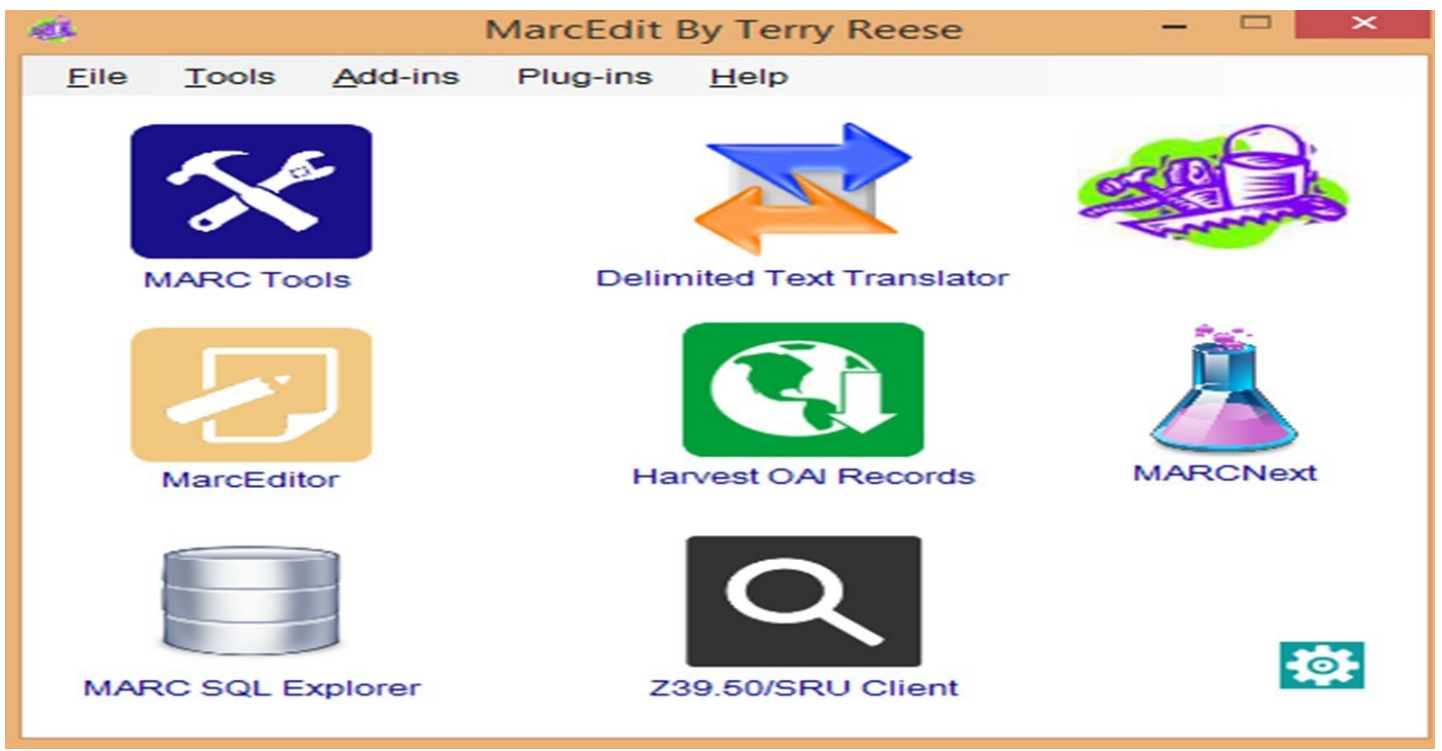

Figura 6. MarcEdit

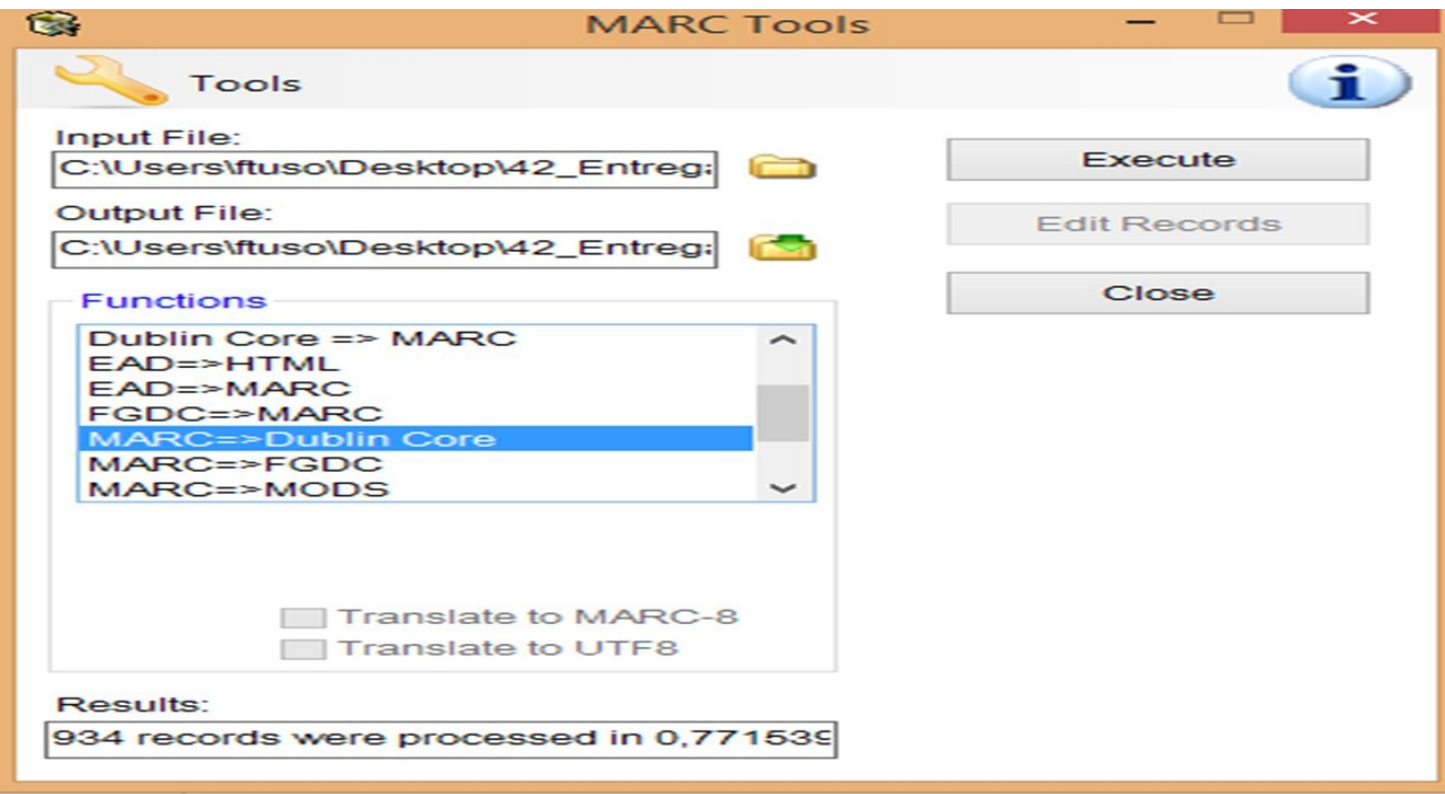

Figura 7. MarcEdit Tools

Los resultados de aplicar las etiquetas mapeadas, de acuerdo al mapeo de MARC a Dublin Core se presentan en la Figura 8: 


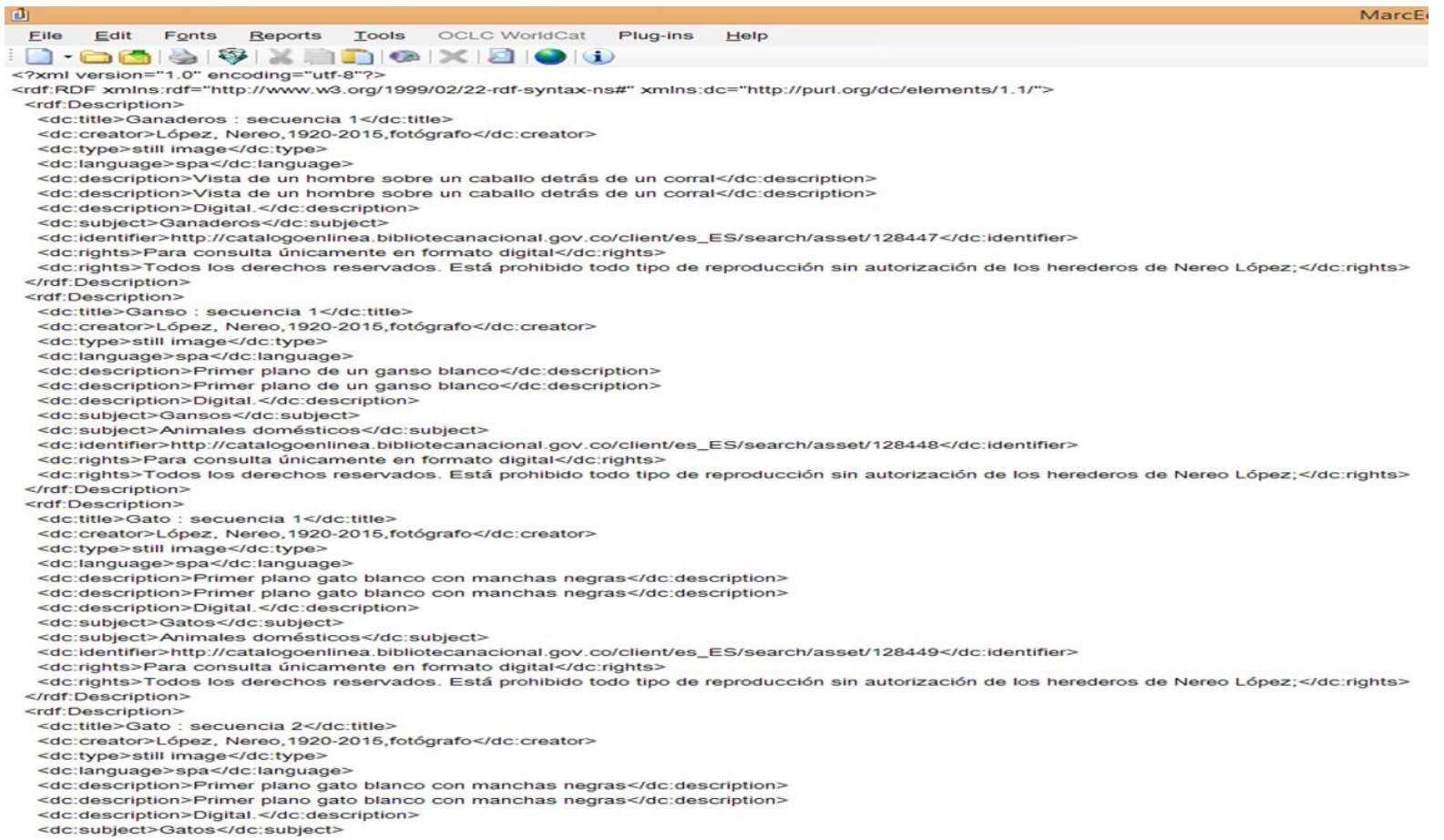

Figura 8. Registros MARC en formato Dublin Core

De esta manera y con la anterior metodología, aplicando la herramienta Marc Edit, se realizan las cargas de los objetos digitales a la herramienta, una vez convertidos y mapeados los datos.

\section{Autoridades RDA / BNC}

Por otro lado, desde la BNC consideramos que la aplicación del estándar en el control de autoridades debe ser desarrollada a nivel país. Por tal motivo, estamos apostando a la creación de un catálogo de autoridades nacional, donde a partir de los cambios de autoridad personal, corporativa y de familia, se permita de manera organizada y completa aplicar el modelo RDA, con el objetivo de visibilizar las autoridades colombianas y sus obras. La metodología planteada para el desarrollo de éstas ha sido la siguiente:

- Migración en formato MARC del SIB Shymphony de la base de datos matriz de catalogación.

- Normalización de registros y unificación de autores.

- Aplicación del estándar y etiquetas RDA de acuerdo al modelo de autoridades personales.

- Migración al repositorio central de autoridades, una vez normalizada la información.

- Integración de las entidades participantes en la prueba piloto, como las universidades, en el desarrollo del catálogo centralizado.

Es así como se ha implementado en la herramienta de software libre KOHA el desarrollo de autoridades personales, incluyéndose en la figura 9 la correspondiente a Gabriel García Márquez. 


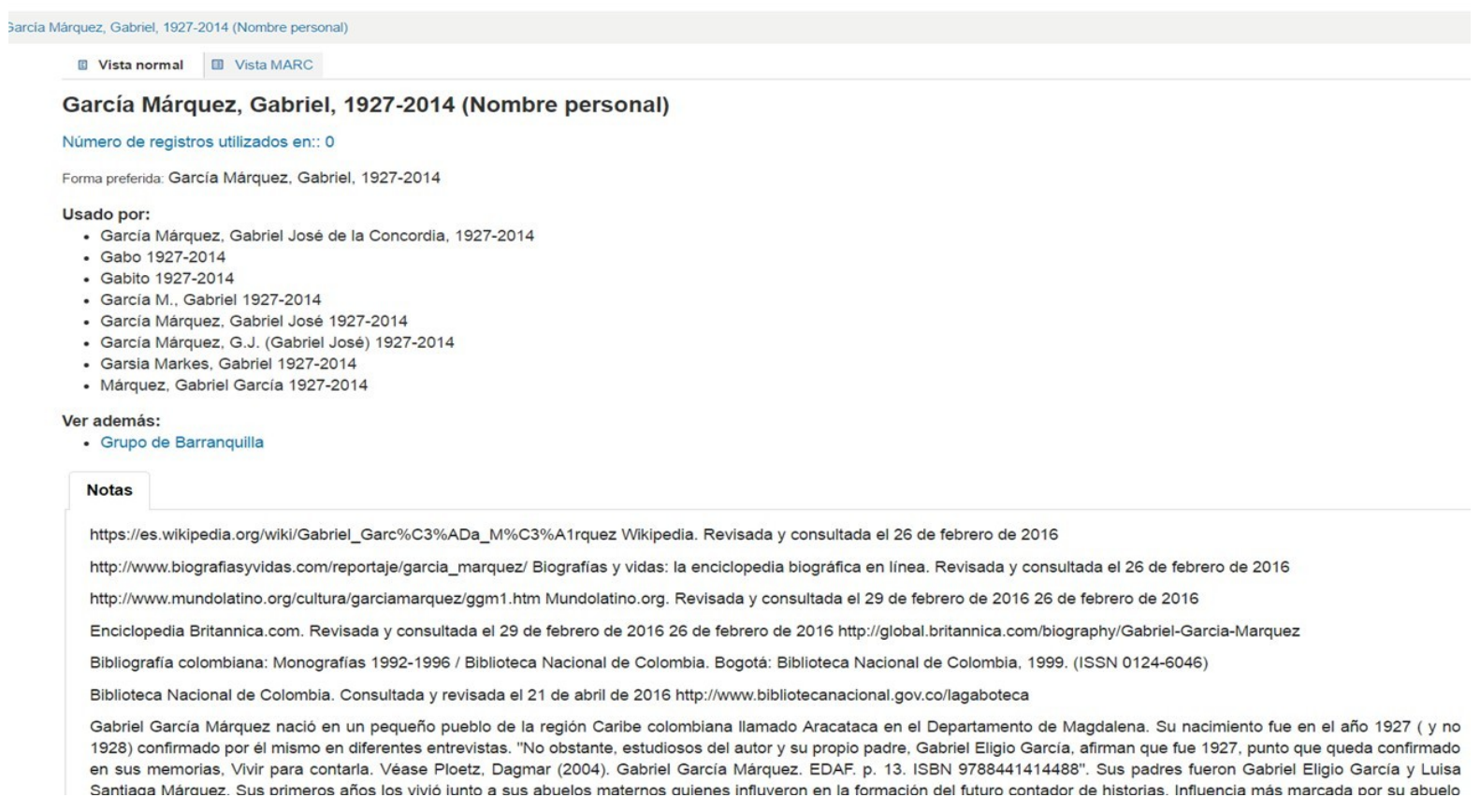

Figura 9. Autoridad Personal (García Márquez, Gabriel) en RDA

Esta metodología ha permitido representar las autoridades en el formato MARC, desplegando los campos RDA de la forma que se muestra en la Figura 10.

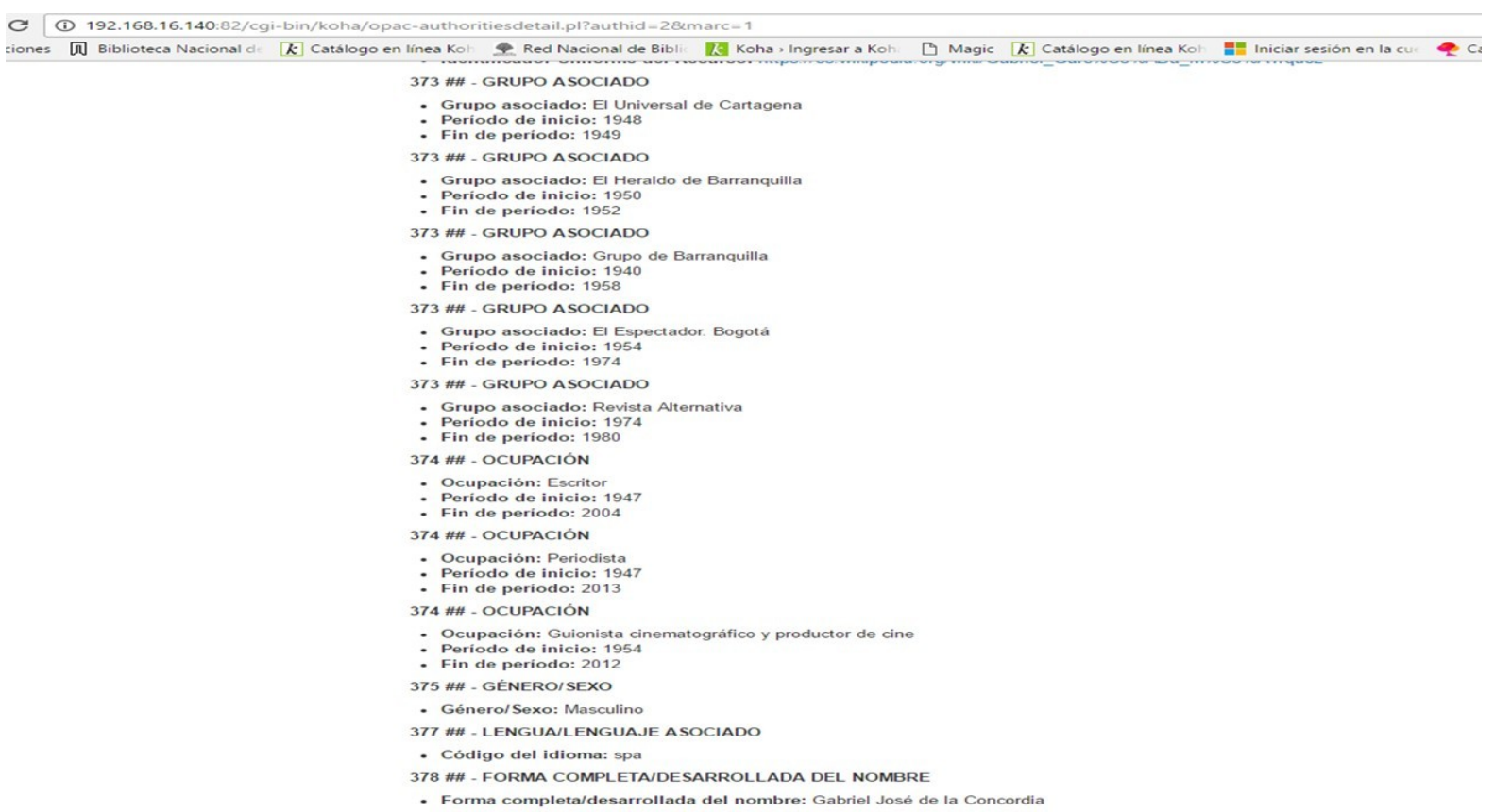

Figura 10. Autoridad Personal en RDA

\section{Conclusiones}

Todo lo expuesto anteriormente permite generar algunas conclusiones. En primera instancia, si el software bibliográfico y los desarrolladores de éste no garantizan la aplicación de las relaciones en los registros bibliográficos y el modelo RDA, podemos decir que no se garantizará 
la correcta implementación de la norma en cualquier unidad de información. Para esto se propone plantear los siguientes interrogantes para el sector bibliotecario latinoamericano y mundial:

1. ¿Realmente desde la bibliotecología estamos trabajando en la implementación del modelo RDA con la industria de software bibliográfico?

2. ¿Realmente necesitamos reemplazar a MARC21?

3. ¿La iniciativa BIBFRAME está de la mano con la industria de software bibliográfico comercial y libre?

4. ¿Cuántas bibliotecas en América Latina han aprobado la implementación de RDA con todos los componentes mencionados?

5. ¿Nuestra participación como bibliotecólogos es activa en la aplicación del modelo RDA con software libre, aplicaciones web, modelos de software bibliográfico, manejando el modelo propuesto y nuevos enfoques como bibliotecarios en el Siglo XXI?

6. ¿Qué tan preparados estamos los bibliotecólogos desde el enfoque técnico y conceptual para proponer el desarrollo de nuevos catálogos a partir del avance de la Web?

7. ¿Bibliotecológicamente estamos desarrollando catálogos digitales y productos digitales en el desarrollo de proyectos relacionados con: obras artísticas, digitalización de fotografías, catálogos especializados, catalogación especializada de objetos audiovisuales, lenguaje de marcado XML, HTML5, mpega21, Dublin Core, entre otros?

8. ¿Cuál será el modelo de metadatos a implementar con el objetivo de garantizar la correcta implementación, que pasará con MARC21?

9. ¿El trabajo de metadatos desarrollado por los catalogadores con la aplicación de RDA es visible en los catálogos y es clara la representación de información para nuestros usuarios?

10.Teniendo en cuenta a Bianchini \& Guerrini (2015): "Dicha transformación se materializa en dos grandes divisiones al interior de RDA. Por un lado, en lo concerniente al registro (descripción) y la visualización y ordenamiento de los datos." ¿Están los actuales catálogos desconectados de las dos grandes divisiones que mencionan estos autores?

\section{Referencias bibliográficas}

Bianchini, C. \& Guerrini, M. (2015). RDA: Resource Description and Access. El nuevo estándar de metadatos y descubrimiento de recursos en la era digital. Información, cultura y sociedad, 33. Recuperado de http://www.redalyc.org/articulo.oa?id $=263042678008$

Biblioteca Nacional de Colombia (2015). La Biblioteca Nacional de Colombia lidera la prueba piloto RDA en el país. bibliotecanacional.gov.co. Recuperado de http://www.bibliotecanacional.gov.co/content/la-biblioteca-nacional-lidera-la-prueba-pilotorda-en-colombia

IFLA (2008). Requisitos Funcionales de los Datos de Autoridad (FRAD): Un modelo conceptual. IFLA. Recuperado de http://www.ifla.org/files/assets/cataloguing/frad/frad 2009-es.pdf 
RDA Steering Committee (2010). RDA Toolkit: Resource Description and Access. rdatoolkit.org. Recuperado de www.rdatoolkit.org

Tillett, B. (2005). ¿Qué es FRBR? Un modelo conceptual del universo bibliográfico. Recuperado de https://www.loc.gov/catdir/cpso/Que-es-FRBR.pdf 